\title{
Black Holes and the Nature of the Expansion of the Universe
}

\author{
Paul Smeulders \\ The Nursery, Sutton Courtenay, UK \\ Email: paul.smeulders@btinternet.com \\ Received 13 January 2016; accepted 21 May 2016; published 24 May 2016 \\ Copyright (C) 2016 by author and Scientific Research Publishing Inc. \\ This work is licensed under the Creative Commons Attribution International License (CC BY). \\ http://creativecommons.org/licenses/by/4.0/

(c) (i) Open Access

\begin{abstract}
Black Holes absorb matter and because matter has structure in space and time, Black Holes absorb the grid points of space and time. It is this property that creates a void for the remaining visible space-time to expand into. This expansion is roughly proportional to the density of the black holes in space and this, because also time is expanding, leads to an apparent exponential expansion for the observer, who is unaware of this. Further the Quantum Mechanical effects: the Heisenberg uncertainty principle and the Tunnelling of particles lead to a flow of matter from the present time into the past. The red-shift is thus not just a sign of an expanding universe but also (and perhaps all of it) that of matter hurling down into the black holes.
\end{abstract}

\section{Keywords}

Expansion of Universe, Black Holes, Speed of Light, Big Bang

\section{Introduction}

It has been shown that the expansion of the universe not only occurs over space but also over time. The clocks over time slow down and the expansion can then be understood to occur over the Lorentz space-time [1].

If one takes a clock that does not change over time then the expansion of the universe seems to be more or less linear in time. This clock is called the true clock. The acceleration of the expansion only seems to occur for the observer since he does not have this kind of clock.

What now makes the universe to expand linearly in true time?

Black holes are known [2] to absorb matter and because of that they absorb its space-time structure. This makes room for the remaining space-time to relax into.

\section{The Black Hole Properties}

Similar things are happening for matter that is falling into a black hole as for an expanding universe. Only it

How to cite this paper: Smeulders, P. (2016) Black Holes and the Nature of the Expansion of the Universe. Journal of Modern Physics, 7, 908-910. http://dx.doi.org/10.4236/jmp.2016.79082 
happens in a sort of reverse way: one has to reverse the time in an expanding universe to get this similarity.

For matter that fall into black holes, the clocks will start to spin up because of the compression of the space coordinates: matter falling into a black hole experiences just the opposite of the matter expanding in an expanding universe: in one case the Lorentz space-time structure is expanding and in the case of matter falling into a black hole the Lorentz space-time structure is being compressed. This can be deduced from equation (8) of ref. [1] replacing the Red Shift $z+1$ by $c(t) /\left(c_{0} \cdot a(t)\right)$. The scale length $a(t)$ is compressed when matter falls into a black hole. The clock rate is increasing. Conservation of angular impulse momentum implies that particles must spin faster and faster when falling into a black hole and that implies that the speed of light in true space-time units must be increasing too. Also the speed of light has to increase because of the increasing clock rate, otherwise the local speed of light will not have its usual local constant value.

Together with this matter, space and time grid points are disappearing into the black holes making room for the space and time grid points of the remaining visible world to expand into.

This expansion of the space grid points will be proportional to the density of the black holes in the universe (so linear in true time if black holes would remain unchanged over time).

And the expansion of the time grid points will create the illusion for the observer that this expansion is exponential [1] (the so-called acceleration of the expansion). A linear expansion of space over true time will lead, if time also expands linearly, to a pure exponential expansion of space as seen by an unaware observer.

\section{Further Properties of Black Holes}

Inside a black hole is most of the space-time structure present that fell into it since its creation, except for the matter that tunnelled out of it in the course of time.

Because of the Heisenberg uncertainty principle all the time points are mixed up thoroughly so that any matter, which has fallen into it, still has a much bigger chance to be tunnelling out [2] of it at much earlier times in the past. On average the tunnelling time should be around half the life-time of the black hole (assuming that all time points have equal chances for tunnelling).

So a black hole could lead to a continuous transport of matter from the present to the past.

Another property of a black hole is that at the inside, the speed of light must be close to infinity because it has to be bigger than the spinning speed of any particle. Remember the speed of light is infinite at the time of the Big Bang [1]. Of course the value of the speed of light in local space and time units is always the same and has its usual value. It is in true time and true space units, that it's value must be close to infinity.

This has implications for the size $R$ of the black hole:

$$
R=\frac{2 G M}{c^{2}}
$$

where $R$ is the event horizon, $G$ is the gravitational constant, $M$ the mass of the black hole and $c$ the speed of light [2].

Clearly the size of any black hole will be very small even if $G$ scales with $c^{3}$ [3] (G.M will scale with $c$, so $R$ will scale as $1 / c)$.

\section{Expansion}

If the observed red-shift can be fully explained by the matter falling into the Black Holes, the question arises if the universe really started with the Big Bang.

Take for instance the Microwave background radiation, which is related to times shortly after the Big Bang. Can this long wavelength radiation be explained by matter falling into the black holes emitting radiation, when the speed of light has already very large values in true space-time units close to the event horizon of the black hole in question. And hence the light quanta there will have very large wavelengths.

At the moment it appears that this is still an open question and that it is not possible to choose between one or the other.

\section{Wormholes}

If Wormholes [2] are connecting Black Holes then it must be that these are of equal mass and have identical 
properties, because the infinite speed of light inside the Black Holes will spread all particles evenly around. So any observation of group of Black Holes of equal mass would support the presence of Wormholes and the infinity of the speed of light inside the Black Holes. One has to take into account possible evolutions of the black holes over time.

The fact that there are many observations of black holes of very different sizes implies that wormholes are not such a frequent occurrence in the universe.

\section{Conclusion}

There is a strong possibility that there is no real expansion of the universe at all and that all the observed red-shift is caused by the matter that is falling continuously into the many black holes present in the universe.

\section{References}

[1] Smeulders, P. (2013) Journal of Modern Physics, 4, 780-783. http://dx.doi.org/10.4236/jmp.2013.46107

[2] Hawking, S. (2001) The Universe in a Nutshell. Bantam Press, New York.

[3] Smeulders, P. (2012) Journal of Modern Physics, 3, 345-349. http://dx.doi.org/10.4236/jmp.2012.34047 